UDC 539

\title{
Schwinger effect for non-Abelian gauge bosons
}

\author{
M. Ragsdale, D. Singleton* \\ Department of Physics, California State University Fresno, Fresno, CA 93740-8031, USA \\ *e-mail:doug@mail.fresnostate.edu
}

\begin{abstract}
We investigate the Schwinger effect for the gauge bosons in an unbroken non-Abelian gauge theory ( e.g. the gluons of QCD). For the simplicity the $S U(2)$ non-Abelian gauge theory is considered. We consider both constant"color electric" fields and "color magnetic" fields as backgrounds. As in the Abelian Schwinger effect we find there is production of "gluons" for the color electric field, but no particle production for the color magnetic field case. Since the non-Abelian gauge bosons are massless there is no exponential suppression of particle production due to the mass of the electron/positron that one finds in the Abelian Schwinger effect. Despite the lack of an exponential suppression of the gluon production rate due to the masslessness of the gluons, we find that the critical field strength is even larger in the non-Abelian case as compared to the Abelian case. The calculations for gluon production from a uniform chromoelectric field and from a uniform chromomagnetic field are made. This is the result of the confinement phenomenon on QCD.
\end{abstract}

PACS: 21.00

Key words: Schwinger effect, gauge bosons, Abelian Schwinger effect, uniform color magnetic field

\section{Introduction}

The Schwinger effect $[1,2]$ is the creation of electron-positron pairs from a uniform electric field. The energy to create the pairs comes from energy of the electric field. Since the rest mass energy of the electron is large relative to the electric field energy density that one can achieve in the laboratory the Schwinger effect has not been observed experimentally in the form in which it was first calculated - a uniform background electric field producing electron-positron pairs. The reason for this is the probability per unit volume per unit time of creating $e^{+} e^{-}$pairs is given by

$$
\frac{\operatorname{prob}_{e^{+} e^{-}}}{\operatorname{Vol} \times \operatorname{time}} \propto e^{2} E_{E M}^{2} \exp \left(-\frac{\pi m^{2}}{e E_{E M}}\right),
$$

where $e$ and $m$ are the charge and mass of the electron/positron, $E_{E M}$ is the magnitude of the uniform electric field and $c$ and $\hbar$ have been set to 1 . Since the electron/positron have a finite rest mass the exponential will suppress $e^{+} e^{-}$production unless $\frac{\pi m^{2}}{e E_{E M}} \sim 1$. If one takes $\frac{\pi m^{2}}{e E_{E M}}=1$, restores factors of $c$ and $\hbar$ then one finds the this uniform electric field magnitude is enormous $-E_{E M}=1.4 \times 10^{14} \frac{\text { dyne }}{\text { esu }}$ or $E_{E M}=4.2 \times 10^{18} \frac{N}{C}-$ well beyond the present ability to create in a laboratory. If electron/positrons were lighter, or massless, one would more readily be able to observe the electromagnetic Schwinger effect.

While the electron/positron mass is non-zero there is a system where a charged particle is massless and thus the Schwinger effect should not have the exponential suppression found in the electromagnetic case. The is the case of gluons in quantum chromodynamics (QCD). Gluons are massless and carry color charge due to the non-Abelian nature of QCD. Thus we want to investigate the QCD version of the Schwinger effect whereby a constant background color "electric" field creates gluons. Of course due to color confinement one can not really make a constant color field over a macroscopic distance. However within QCD bound systems one might think of the quantum chromodynamic flux tubes that are postulated to bind quarks together into mesons/baryons as giving a uniform color electric and color magnetic fields inside the tubes.

In this work we consider, for simplicity, the $S U(2)$ non-Abelian gauge theory. This has almost all of the features of larger non-Abelian groups like $S U(3)$ but the details of the calculations are simpler and more transparent. Often in this work we will mention "gluons" or QCD which technically refer to the strong $S U(3)$ gauge group, but we mean by this the $S U(2)$ toy model of the true $S U(3)$ interaction. 


\section{Brief review of electric Schwinger effect}

In this section we will give a very brief overview of Schwinger's method for calculating pair production in the case of Abelian electromagnetic fields. In the following section we will use this background to address pair production in a nonAbelian color electric and color magnetic fields. In addition to the original articles on the Schwinger effect $[1,2]$ there are many good discussion of this method in the literature. A very small sample of these are found in references $[3,4,5,6]$. We will follow most closely the pedagogical article [3].

To begin the vacuum to vacuum transition amplitude is given very generally by the expression

$$
\operatorname{amp}(v a c \rightarrow v a c) \rightarrow \int[d \phi] \exp \left(i \int d^{4} x L\left(\phi, \partial_{\mu} \phi\right)\right),
$$

where $\phi$ is some generic field, $\int[d \phi]$ is a path integral over different field configurations, and $L\left(\phi, \partial_{\mu} \phi\right)$ is the Lagrange density for the field $\phi$. The Lagrange density will lead to an equation of motion for the field $\phi(x)$ in terms of some operator $O \phi$. The field $\phi(x)$ can be expanded as $\phi(x)=$ $\sum_{n} a_{n} \chi_{n}(x)$ where $\chi_{n}(x)$ are eigenstates of the equation of motion given by

$$
O \chi_{n}(x)=\lambda_{n} \chi_{n}(x)
$$

with $\lambda_{n}$ being the eigenvalues. In terms of the operator $O$ the vacuum to vacuum amplitude is $\operatorname{amp}(v a c \rightarrow v a c) \sim \frac{\text { const. }}{\operatorname{deto}}$ where the determinant of the operator can be written in terms the eigenvalues as $\operatorname{det} O=\prod_{n} \lambda_{n}$. Using all this we can write out the results as

$$
\begin{gathered}
\frac{1}{\operatorname{det} O}=\exp [-\ln (\operatorname{det} O)]= \\
=\exp \left[-\ln \prod_{n} \lambda_{n}\right]= \\
=\exp \left[-\sum_{n} \ln \lambda_{n}\right]=\exp [-\operatorname{tr} \ln (O)] .
\end{gathered}
$$

Next we use the representation of the logarithm as $\ln \lambda_{n}=-\int_{0}^{\infty} \frac{\exp \left(-\lambda_{n} s\right)}{s} d s$ to write the middle expression in(4) as

$$
\operatorname{tr}(\ln O)=-\sum_{n} \int_{0}^{\infty} \frac{\exp \left(-\lambda_{n} s\right)}{s} d s \equiv \zeta .
$$

Finally the vacuum to vacuum amplitude from (2) becomes

$$
\operatorname{amp}(v a c \rightarrow v a c) \propto \exp [-\zeta] .
$$

If $\zeta$ has a real part (i.e. $\gamma=\operatorname{Re}(\zeta)$ ) then one can square the amplitude to get the probability for pair production as

$$
\text { prob }_{\text {pair }}=(1-\exp [-2 \gamma]) \text {. }
$$

This is the basic procedure which we will apply to the pair production of gluons in uniform color electric and color magnetic fields. However before moving on to this we give a few more details about the pair production of scalar particles of mass $m$ and charge $e$ in a uniform electric field. For a uniform electric field in the $z$-direction $E=E_{0} \hat{z}$ the vector potential can be of the form $A(t)=-E_{0} t \hat{z}$ or $\phi(z)=$ $-E_{0} z$. (In the QCD case we will find a similar situation for the QCD potentials). For the timedependent vector potential, $A(t)=-E_{0} t \hat{z}$, the operator in footnote 1 becomes

$$
O=\partial_{t}^{2}-\left(\partial_{z}-i e E_{0} t\right)^{2}-\partial_{x}^{2}-\partial_{y}^{2}+m^{2} .
$$

The eigenvalues connected with (8) are

$$
\begin{gathered}
\lambda_{n}=e E_{0}(2 n+1)+p_{x}^{2}+ \\
\quad+p_{y}^{2}+m^{2}, \mathrm{n}=0,1,2 \ldots .
\end{gathered}
$$

Using this eigenvalues in (5) and performing the sum of the different pieces of $\lambda_{n}$ yields

$$
\zeta=-i L^{3} T \frac{e E_{E M}}{16 \pi^{2}} \int_{0}^{\infty} \frac{d s}{s^{2}} \frac{\exp \left[-m^{2} s\right]}{\sin \left(e E_{E M} s\right)^{\prime}}
$$

where $L$ and $T$ are the spatial and temporal size to "cube" inside which the system is quantized. The factor of $i$ comes from doing a rotation to imaginary time and back (i.e. $t \rightarrow i T$ ). This time rotation also 
involves the change $E_{E M} \rightarrow i E_{E M}$. We will use the same procedure when we carry out the QCD version of this calculation. In order to have particle production $\zeta$ needs to have a real part which will occur if the integral in (10) has an imaginary part. The integral in (10) does have imaginary parts coming from the contour integrations involving the poles in the integrand at $s_{n}=n \pi / e E_{E M}$. (The $1 / s^{2}$ divergence in the integrand in (10) leads to an infinite imaginary part which is removed via renormalization [7]). Taking semi-circular deviations around each of the poles $s_{n}$ leads to a real part of $\zeta$ given by

$$
\gamma=\operatorname{Re}(\zeta)=L^{3} T \frac{e^{2} E_{E M}^{2}}{16 \pi^{3}} \sum_{n=1}^{\infty} \frac{(-1)^{n+1}}{n^{2}} \exp \left(-\frac{\pi n m^{2}}{e E_{E M}}\right) \approx L^{3} T \frac{e^{2} E_{E M}^{2}}{16 \pi^{3}} \exp \left(-\frac{\pi m^{2}}{e E_{E M}}\right) .
$$

Due to the exponential term the main contribution comes from $n=1$ term in the sum. The result in (11) can be used to obtain the result in (1) by inserting it into (7) and dividing by $(\mathrm{Vol} \times$ time $)$

$$
\frac{\text { prob }_{e^{+} e^{-}}}{\text {Vol } \times \text { time }}=\frac{1}{\text { Vol } \times \text { time }}\left(1-e^{-2 \gamma}\right) \approx \frac{e^{2} E_{E M}^{2}}{8 \pi^{3} \hbar^{2} c} \exp \left(-\frac{\pi m^{2} c^{3}}{e E_{E M} \hbar}\right)=\frac{\alpha_{E M} E_{E M}^{2}}{8 \pi^{3} \hbar} \exp \left(-\frac{\pi m^{2} c^{3}}{e E_{E M} \hbar}\right),
$$

where we have restored factors of $\hbar$ and $c$ and then written the result in terms of the fine structure constant $\alpha_{E M}=\frac{e^{2}}{\hbar c} \approx \frac{1}{137}$. We want to use (12) to determine prob $_{e^{+}} e^{-}$when the electric field is at the critical value $E_{E M} \approx 1.4 \times 10^{14} \frac{d y n e}{e s u}$. To do this we need some way to characterize what a "natural" value is for the volume and time in the denominator of (12). For the volume we take it to be the cube of the reduced Compton wave length of the electron $\mathrm{Vol}=$ $\left(\frac{\hbar}{m c}\right)^{3} \approx 5.7 \times 10^{-32} \mathrm{~cm}^{3}$. For the time we take the reduced Compton time time $=\frac{\hbar}{m c^{2}} \approx 1.3 \times$ $10^{-21}$ sec. Putting all these values together in (12) yields

$$
\operatorname{prob}_{e^{+} e^{-}} \approx 0.015
$$

In the next section we will calculate the equivalent result to equation (12) but for gluons produced from uniform chromoelectric fields and uniform chromomagnetic fields. We will set the probability of gluon production (i.e. $\operatorname{prob}_{g g}$ ) to the same value as that of $e^{+} e^{-}$production from (13) for the critical electric field, $E_{E M}$. In this way we will arrive at the critical chromoelectric/chromomagnetic field strength to produce gluons. The initial expectation might be that the critical color field strength might be smaller since gluons are massless so there will be no exponential suppression as occurs due to the rest mass of the electron for the QED case.

\section{Schwinger effect for uniform color electric field and uniform color magnetic field}

To calculate the production rate for gluons from uniform chromoelectric and chromomagnetic fields we follow reference [8] and place gluon field excitations in a fixed background field. For the two background fields we take a uniform chromoelectric field and uniform chromomagnetic field. These calculations are rather involved and we put the details in appendix 1 for the uniform chromoelectric field and Appendix 2 for the uniform chromomagnetic field. The result for the production rate for gluons in a uniform chromoelectric field is given by equation (42) as

$$
\gamma \approx 0.00187 \times L^{3} T \frac{g^{2} E_{0}^{2}}{16 \pi^{3}} .
$$

Using this result from (14) in (7) we obtain the probability for $\mathrm{SU}(2)$ gluon creation per unit volume and unit time

$$
\begin{gathered}
\frac{\text { prob }_{g g}}{\text { Vol } \times \text { time }}=\frac{1}{\text { Vol } \times \text { time }}\left(1-e^{-2 \gamma}\right) \approx \\
\approx \frac{0.00187 g^{2} E_{0}^{2}}{8 \pi^{3} \hbar^{2} c}=\frac{0.00187 \alpha_{Q C D} E_{0}^{2}}{8 \pi^{3} \hbar} .
\end{gathered}
$$

In the last step we have restored factors of $\hbar$ and $c$ and introduced the QCD fine structure constant $\alpha_{Q C D}=\frac{g^{2}}{\hbar c}$. We will take $\alpha_{Q C D} \approx 1$ so that we are in non-perturbative regime. For the present case we take 
the distance scale to be $10^{-12} \mathrm{~cm}$. This is one order of magnitude larger than the typical strong interaction bound state size of $1 \mathrm{fermi}$. Thus is the volume factor in (15) we have $\mathrm{Vol}=10^{-36} \mathrm{~cm}^{3}$ and for the time factor we take time $=\frac{10^{-12}}{3 \times 10^{10}}=3.3 \times$ $10^{-23} \mathrm{sec}$. Finally we take the value for the probability of gluon production, prob $_{g g}$ at the critical chromoelectric field magnitude to be the same as that for the electromagnetic case as given in (13) namely $\operatorname{prob}_{g g} \approx 0.015$, using these assumptions in (15) we can calculate the value of the critical chromoelectric field magnitude as

$$
E_{0} \approx 2.5 \times 10^{17} \frac{\mathrm{d} y n e}{\mathrm{esu}} .
$$

This is then three orders of magnitude larger than the $E \& M$ critical field value of $E_{E M} \approx 1.4 \times$ $10^{14} \frac{d y n e}{\text { esu }}$. Thus although gluons are massless (and thus there is no exponential suppression factor as in the electromagnetic case due to the mass of the electron) this does not lower the critical chromoelectric field value, and in fact we find the critical field value for QCD is three orders of magnitude larger than in the $E \& M$ case. This, at first sight, surprising result arises from the fact that QCD has a confinement scale in the range of 1 fermi $=$ $10^{-13} \mathrm{~cm}$. This distance is smaller than the Compton wave length of the electron which we used to set the volume and time in the electromagnetic case as $\mathrm{Vol}=\left(\frac{\hbar}{m c}\right)^{3} \approx 5.7 \times 10^{-32} \mathrm{~cm}^{3}$ and time $=\frac{\hbar}{m c^{2}} \approx$ $1.3 \times 10^{-21}$ sec respectively. In the QCD case we overestimated the distance scale as 10 times larger than 1 fermi $=10^{-13} \mathrm{~cm}$ and also used this to obtain the characteristic time for the QCD case. Even though we (slightly) overestimated the QCD distance and time scales (which would by (15) would be favorable toward to decreasing the critical QCD field strength) we nevertheless found that the critical QCD field strength was larger than the critical $E \& M$ critical field strength. Even if we had used (without justification) the electron Compton wave length and associated Compton time in (15) this would give a QCD critical field strength of $E_{0} \sim 10^{14} \frac{\text { dyne }}{\text { esu }}$ i.e. the same as for $E \& M$. Thus the masslessness of the gluons does not lead to a lower critical field strength relative to what is found in the canonical Schwinger mechanism case.

\section{Conclusions}

We presented the Schwinger effect for SU(2) gluons. We did this since gluons being massless we expect this effect to be more important than standard Schwinger effect which is exponentially suppressed due to electron/positron rest mass. The calculation details for gluon production from a uniform chromoelectric field and from a uniform chromomagnetic field are found in Appendix A and $\mathrm{B}$ respectively. As in the electromagnetic case a chromoelectric field will produce gluons while the chromomagnetic field does not.

The motivation for investigating gluon production, as opposed to the production of quarks, was that since gluons are massless there will not be an exponential suppression due to the mass of the quarks that one finds in the electromagnetic case due to the mass of the electron (i.e. the last exponential term in (1)). However, due to confinement the natural length scales and time scale for the QCD case were several orders of magnitude smaller than the similar scales for the electromagnetic case which were set to the reduced Compton length of the electron and the associated time. This in turn made the factor $\mathrm{Vol} \times$ time smaller for the QCD case as compared to the $E \& M$ case which in turn, given the expression for the probability for production per unit volume per unit time in (15) gave a larger magnitude for the chromoelectric field for producing gluons as compared to the magnitude of the electric field for producing electrons and positrons.

\section{Acknowledgment}

DS is supported by grant $\Phi .0755$ in fundamental research in Natural Sciences by the Ministry of Education and Science of Kazakhstan.

\section{Appendix I: Uniform Color Electric Field Calculation}

To have a constant color electric field in the $\hat{z}=$ 3 spatial direction and in the $a=3$ color direction,

$$
\begin{aligned}
& E_{\mu}^{a} \rightarrow E_{3}^{3}=F_{03}^{3}=\partial_{0} A_{3}^{3}- \\
- & \partial_{3} A_{0}^{3}+g \varepsilon^{3 b c} A_{3}^{b} A_{0}^{c}=E_{0} \hat{z},
\end{aligned}
$$

there are two gauge choices one can make for the potential 


$$
\text { (i) } A_{\mu}^{a}=E_{0} t \delta_{\mu 3} \delta^{a 3} ;(i i) A_{\mu}^{a}=-E_{0} z \delta_{\mu 0} \delta^{a 3} .
$$

For the calculation in this appendix we use the $(i)$ form of the potential. Inserting form $(i)$ from (18) into (17) does give $E_{\mu}^{a} \rightarrow E_{3}^{3}=\partial_{0} A_{3}^{3}=E_{0} \hat{z}$. We now take the potential $(i)$ from (18) as a background potential (i.e. $A_{\mu}^{(0) a}$ ) and we consider small variation $A_{v}^{a}$ around this background (the background nature of the potential is indicated by the superscript (0)). In this way the QCD action can be written as

$$
F_{\mu \nu}^{a} F^{a \mu \nu}=\left[\begin{array}{c}
\left(\partial_{\mu} A_{\nu}^{(0) a}-\partial_{\nu} A_{\mu}^{(0) a}\right)+\left(\partial_{\mu} A_{\nu}^{a}-\partial_{\nu} A_{\mu}^{a}\right) \\
+g \varepsilon^{a b c}\left(A_{\mu}^{(0) b}+A_{\mu}^{b}\right)\left(A_{\nu}^{(0) c}+A_{\nu}^{c}\right)
\end{array}\right]^{2}
$$

We now run through the color indices $a=1,2,3$ and insert the explcit form of the background potential $A_{\mu}^{(0) 3}=E_{0} t \delta_{\mu 3}$. The only non-zero values of $\partial_{\mu} A_{\nu}^{(0) 3}-\partial_{\nu} A_{\mu}^{(0) 3}+g \varepsilon^{3 b c} A_{\mu}^{(0) b} A_{\nu}^{(0) c}$ are when $\mu=0$ and $v=3$ or vice versa, each of which contribute a term of $E_{0}$.

$$
\begin{gathered}
F_{\mu \nu}^{a} F^{a \mu \nu}=\left\{\partial_{\mu} A_{v}^{1}-\partial_{v} A_{\mu}^{1}+g\left[A_{\mu}^{2}\left(A_{v}^{3}+E_{0} t \delta_{v 3}\right)-\left(A_{\mu}^{3}+t \delta_{\mu 3}\right) A_{v}^{2}\right]\right\}^{2} \\
+\left\{\partial_{\mu} A_{v}^{2}-\partial_{\nu} A_{\mu}^{2}+g\left[\left(A_{\mu}^{3}+E_{0} t \delta_{\mu 3}\right) A_{v}^{1}-A_{\mu}^{1}\left(A_{v}^{3}+E_{0} t \delta_{v 3}\right)\right]\right\}^{2} \\
+\left[\partial_{\mu} A_{v}^{3}-\partial_{\nu} A_{\mu}^{3}+g\left(A_{\mu}^{1} A_{v}^{2}-A_{\mu}^{2} A_{v}^{1}\right)\right]^{2} \\
+2 E_{0}^{2}-2 E_{0}\left[\partial_{\mu} A_{v}^{3}-\partial_{\nu} A_{\mu}^{3}+g\left(A_{\mu}^{1} A_{v}^{2}-A_{\mu}^{2} A_{v}^{1}\right)\right]\left(\delta_{\mu 3} \delta_{\nu 0}-\delta_{\mu 0} \delta_{\nu 3}\right)
\end{gathered}
$$

We now expand the above expression to $2^{\text {nd }}$ order in the $A_{\mu}^{a}$ which gives

$$
\begin{gathered}
F_{\mu \nu}^{a} F^{a \mu \nu} \approx \\
\approx\left[\partial_{\mu} A_{\nu}^{1}-\partial_{\nu} A_{\mu}^{1}-g E_{0} t\left(A_{\nu}^{2} \delta_{\mu 3}-A_{\mu}^{2} \delta_{v 3}\right)\right]^{2} \\
+\left[\partial_{\mu} A_{\nu}^{2}-\partial_{\nu} A_{\mu}^{2}-g E_{0} t\left(A_{\mu}^{1} \delta_{v 3}-A_{\nu}^{1} \delta_{\mu 3}\right)\right]^{2} \\
+\left(\partial_{\mu} A_{\nu}^{3}-\partial_{\nu} A_{\mu}^{3}\right)^{2}+2 E_{0}^{2}- \\
4 E_{0}\left[\partial_{3} A_{0}^{3}-\partial_{0} A_{3}^{3}+g\left(A_{3}^{1} A_{0}^{2}-A_{3}^{2} A_{0}^{1}\right)\right] .
\end{gathered}
$$

We now require that at spatial and temporal infinity the variation of the potential goes to zero $A_{0}^{3}\left(x_{3} \rightarrow \pm \infty\right)=0$ and $A_{3}^{3}\left(x_{0} \rightarrow \pm \infty\right)=0$. This causes the source terms for $A_{\mu}^{3}$ in the last term to vanish by partial integration. Note that $\left(\partial_{\mu} A_{v}^{3}-\right.$ $\left.\partial_{\nu} A_{\mu}^{3}\right)^{2}$ is a free Lagrange density, so it is not of interest and can be dropped.

$$
\begin{gathered}
F_{\mu \nu}^{a} F^{a \mu \nu}= \\
=\left[\partial_{\mu} A_{v}^{1}-\partial_{\nu} A_{\mu}^{1}-g E_{0} t\left(A_{v}^{2} \delta_{\mu 3}-A_{\mu}^{2} \delta_{\nu 3}\right)\right]^{2} \\
+\left[\partial_{\mu} A_{v}^{2}-\partial_{\nu} A_{\mu}^{2}-g E_{0} t\left(A_{\mu}^{1} \delta_{v 3}-A_{\nu}^{1} \delta_{\mu 3}\right)\right]^{2} \\
+2 E_{0}^{2}-4 E_{0} g\left(A_{3}^{1} A_{0}^{2}-A_{3}^{2} A_{0}^{1}\right) .
\end{gathered}
$$

We now re-write this using complex potential forms as

$$
\begin{aligned}
& F_{\mu \nu}^{a} F^{a \mu \nu}=\left[\begin{array}{c}
\partial_{\mu}\left(A_{v}^{1}+i A_{v}^{2}\right)-\partial_{v}\left(A_{\mu}^{1}+i A_{\mu}^{2}\right) \\
-g E_{0} t\left[\left(A_{v}^{2}-i A_{v}^{1}\right) \delta_{\mu 3}-\left(A_{\mu}^{2}-i A_{\mu}^{1}\right) \delta_{v 3}\right]
\end{array}\right]^{2} \\
& \quad+2 E_{0}^{2}-i 2 g E_{0}\left[\left(A_{3}^{1}+i A_{3}^{2}\right)\left(A_{0}^{1}-i A_{0}^{2}\right)-\left(A_{0}^{1}+i A_{0}^{2}\right)\left(A_{3}^{1}-i A_{3}^{2}\right)\right] .
\end{aligned}
$$

We now replace $A_{\mu}^{1}$ and $A_{\mu}^{2}$ by defining the following complex potetnials

$$
\begin{aligned}
& W_{\mu}=\frac{1}{\sqrt{2}}\left(A_{\mu}^{1}+i A_{\mu}^{2}\right), \\
& W_{\mu}^{\dagger}=\frac{1}{\sqrt{2}}\left(A_{\mu}^{1}-i A_{\mu}^{2}\right) .
\end{aligned}
$$

In terms of these new, complex potentials $F_{\mu \nu}^{a} F^{a \mu \nu}$ becomes

$$
\begin{aligned}
F_{\mu \nu}^{a} F^{a \mu \nu}= & \mid \sqrt{2} \partial_{\mu} W_{v}-\sqrt{2} \partial_{\nu} W_{\mu}-i \sqrt{2} g E_{0} t W_{\nu} \delta_{\mu 3} \\
& +\left.i \sqrt{2} g E_{0} t W_{\mu} \delta_{\nu 3}\right|^{2}+2 E_{0}^{2}- \\
& -i 2 g E_{0}\left(2 W_{3}^{\dagger} W_{0}-2 W_{0}^{\dagger} W_{3}\right) .
\end{aligned}
$$


This finally leads to the following Lagrange density for gluons in the background potential of a uniform color electric field

$$
\begin{gathered}
\mathcal{L}=-\frac{1}{4} F_{\mu \nu}^{a} F^{a \mu \nu} \\
=-\frac{1}{2} \mid\left(\partial_{\mu}-i g E_{0} t \delta_{\mu 3}\right) W_{v} \\
-\left.\left(\partial_{v}-i g E_{0} t \delta_{v 3}\right) W_{\mu}\right|^{2} \\
-i g E_{0}\left(W_{3}^{\dagger} W_{0}-2 W_{0}^{\dagger} W_{3}\right)-\frac{1}{2} E_{0}^{2} .
\end{gathered}
$$

This Lagrange density leads to the following equation of motion

$$
\begin{gathered}
\left(\partial^{\mu}-i g E_{0} t \delta^{\mu 3}\right)\left[\left(\partial_{\mu}-i g E_{0} t \delta_{\mu 3}\right) W_{v}\right. \\
\left.-\left(\partial_{v}-i g E_{0} t \delta_{v 3}\right) W_{\mu}\right]- \\
-i g E_{0}\left(\delta_{v 3} W_{0}-\delta_{v 0} W_{3}\right)=0 .
\end{gathered}
$$

Choosing the background gauge condition $\left(\partial^{\mu}-\right.$ $\left.i g E_{0} t \delta^{\mu 3}\right) W_{\mu}=0$ simplifies (27) to

$$
\begin{gathered}
\left(\partial^{\mu}-i g E_{0} t \delta^{\mu 3}\right)\left(\partial_{\mu}-i g E_{0} t \delta_{\mu 3}\right) W_{v}= \\
-2 i g E_{0}\left(W_{0} \delta_{v 3}-W_{3} \delta_{v 0}\right) .
\end{gathered}
$$

The above equation can be written in matrix form as

$$
\left(\partial^{\mu}-i g E_{0} t \delta^{\mu 3}\right)^{2} W_{v}-2 g E_{0}\left(\begin{array}{cccc}
0 & 0 & 0 & -i \\
0 & 0 & 0 & 0 \\
0 & 0 & 0 & 0 \\
i & 0 & 0 & 0
\end{array}\right) W_{\mu}=0
$$

The four eigenvalues for this matrix are \pm 1 and a double eigenvalue of zero. The zero eigenvalues are excluded by our chosen gauge condition so for now we consider just the eigenvalues \pm 1 . Thus (29) becomes

$$
\left[\left(\partial^{\mu}-i g E_{0} t \delta^{\mu 3}\right)^{2} W_{v}-2 g E_{0}( \pm 1)\right] W_{v}=0 .
$$

Recalling that we are using the metric signature $(+,-,-,-)(30)$ can be expanded as

$$
\left\{\frac{\partial^{2}}{\partial t^{2}}-\frac{\partial^{2}}{\partial x_{1}^{2}}-\frac{\partial^{2}}{\partial x_{2}^{2}}+\left(i \frac{\partial}{\partial x_{3}}+g E_{0} t\right)^{2} \mp 2 g E_{0}\right\} W_{v}=0 .
$$

Next we Fourier transform (31) to $\widetilde{W}_{v}\left(t, k_{1}, k_{2}, k_{3}\right)$ with the result

$$
\left\{\frac{\partial^{2}}{\partial t^{2}}+k_{1}^{2}+k_{2}^{2}+g^{2} E_{0}^{2}\left(\frac{k_{3}}{g E_{0}}+t\right)^{2} \pm 2 g E_{0}\right\} \widetilde{W}_{v}=0 .
$$

Now making the substitution $t^{\prime}=\frac{k_{3}}{g E_{0}}+t$ (for which one has $\left.\frac{\partial}{\partial t}=\frac{\partial}{\partial t}\right)$ and then performing

a rotaion to imaginary time, $t^{\prime} \rightarrow-i \tau, E_{0} \rightarrow-i E_{0}$ we arrive at

$$
\left\{-\frac{\partial^{2}}{\partial \tau^{2}}+g^{2} E_{0}^{2} \tau^{\prime 2}+k_{1}^{2}+k_{2}^{2} \pm 2 i g E_{0}\right\} \widetilde{W}_{v}=0 .
$$

The first two terms in (33) correspond to a harmonic oscillator with frequency $\omega=g E_{0}$, which has eigenvalues given by $-\frac{\partial}{\partial \tau^{2}}+g^{2} E_{0}^{2} \tau^{2} \rightarrow 2(n+$ $\left.\frac{1}{2}\right) g E_{0}$. With this (33) becomes $\left\{(2 n+1) g E_{0}+\right.$ $\left.k_{1}^{2}+k_{2}^{2} \pm i g E_{0}\right\} \widetilde{W}_{v}=0$. From this equation we can read of the eigenvalues as

$$
\lambda_{n}=(2 n+1) g E_{0}+k_{1}^{2}+k_{2}^{2} \pm 2 i g E_{0}
$$

Note that $\lambda_{n}$ is a combination of discrete (i.e. $\left.(2 n+1) g E_{0}\right)$ and continuous (i.e. $\left.k_{1}^{2}+k_{2}^{2}\right)$ parts. Substituting these eigenvalues from (34) into (5) we find that $\zeta$ becomes 


$$
\zeta=-\sum_{n} \int_{0}^{\infty} \frac{\mathrm{d} s}{s} \exp \left\{-\left[(2 n+1) g E_{0}+k_{1}^{2}+k_{2}^{2} \pm 2 i g E_{0}\right] s\right\}
$$

We take our system to be quantized in a cubical spatial volume with sides of length $L$ and over a total (imaginary) interaction time $\tau=-i T$ which then turns (35) into

$$
\zeta=-L \int_{-\infty}^{\infty} \frac{\mathrm{d} k_{1}}{(2 \pi)} L \int_{-\infty}^{\infty} \frac{\mathrm{d} k_{2}}{(2 \pi)} L \int_{0}^{g E_{0} \tau} \frac{\mathrm{d} k_{3}}{(2 \pi)} \sum_{n=0}^{\infty} \int_{0}^{\infty} \frac{\mathrm{d} s}{s} \exp \left\{-\left[(2 n+1) g E_{0}+k_{1}^{2}+k_{2}^{2} \pm 2 i g E_{0}\right] s\right\}
$$

The $k_{1}$ and $k_{2}$ integrations related to the momentum in the free directions and are simple Gaussian integrals, which give two factors of $\sqrt{\frac{\pi}{s}}$. The integration in the $k_{3}$ direction is related to the momentum in the $z$ direction which is the direction of the chromoelectric field. Thus as in [3] this integration is constrained to the range $0<k_{3}<$ $g E_{0} \tau$. Performing the $k_{1}, k_{2}, k_{3}$ integrations gives

$$
\zeta=-L^{3} \tau \frac{g E_{0}}{8 \pi^{2}} \sum_{n=0}^{\infty} \int_{0}^{\infty} \frac{\mathrm{d} s}{s^{2}} \exp \left\{-\left[(2 n+1) g E_{0} \pm 2 i g E_{0}\right] s\right\}
$$

We note that $\sum_{n=0}^{\infty} e^{-(2 n+1) g E_{0} s}=\frac{1}{2} \frac{1}{\sinh \left(g E_{0} s\right)}$ which then transforms (37) into

$$
\zeta=-L^{3} \tau \frac{g E_{0}}{16 \pi^{2}} \int_{0}^{\infty} \frac{\mathrm{d} s}{s^{2}} \frac{e^{ \pm 2 i g E_{0} s}}{\sinh \left(g E_{0} s\right)} .
$$

We now return to real time via the rotation $\tau \rightarrow$ $i T$ which also involves changing the magnitude of the chromoelectric field as $E_{0} \rightarrow i E_{0}$. This gives

$$
\zeta=-i L^{3} T \frac{g E_{0}}{16 \pi^{2}} \int_{0}^{\infty} \frac{\mathrm{d} s}{s^{2}} \frac{e^{\mp 2 g E_{0} s}}{\sin \left(g E_{0} s\right)} .
$$

Equation (39) is now at the point in the electromagnetic calculation given by equation (10), but now the exponential factor involving the electron mass $\left(\exp \left[-m^{2} s\right]\right)$ is replaced by an exponential suppression involving the field strength $\left(e^{\mp 2 g E_{0} s}\right)$. As before if $\zeta$ has a real part (i.e. if the integral in (39) has an imaginary contribution) there will be particle production. As in the case of the electromagnetic integral in (10) the integral in (39) does have an imaginary contribution coming from the poles of $\frac{1}{\sin \left(g E_{0} s\right)}$ whihc are located at $s_{n}=\frac{n \pi}{g E_{0}}$, where $n$ is an integer. As before we ignore the singularity at $s=0$. The integration contours are infinitesimal semicircular in the upper half plane and from (39) this gives

$$
\begin{aligned}
\gamma= & \operatorname{Re}(\zeta)=-i L^{3} T \frac{g E_{0}}{16 \pi^{2}} \sum_{n=1}^{\infty} \int_{s_{n}-\varepsilon}^{s_{n}+\varepsilon} \frac{\mathrm{d} s}{s^{2}} \frac{e^{\mp 2 g E_{0} s}}{\sin \left(g E_{0} s\right)}= \\
= & -i L^{3} T \frac{g E_{0}}{16 \pi^{2}} \sum_{n=1}^{\infty} \int_{s_{n}-\varepsilon}^{s_{n}+\varepsilon} \frac{\mathrm{d} s}{s^{2}} \frac{e^{\mp 2 g E_{0} s}}{\cos \left(g E_{0} s_{n}\right) g E_{0}\left(s-s_{n}\right)}
\end{aligned}
$$

In the last step we have expanded $\sin \left(g E_{0} s\right)$ around the poles at $s_{n}$. From the Residue theorem, the poles of the integral in (40) give $-i \pi \times$ $\sum$ Res(function), where the sum is over the residue of the integrand. The result is

$$
\begin{gathered}
\gamma=\operatorname{Re}(\zeta)=-L^{3} T \frac{1}{16 \pi} \sum_{n=1}^{\infty} \frac{e^{\mp 2 g E_{0} s_{n}}}{s_{n}^{2} \cos \left(g E_{0} s_{n}\right)}= \\
=L^{3} T \frac{g^{2} E_{0}^{2}}{16 \pi^{3}} \sum_{n=1}^{\infty}(-1)^{n+1} \frac{e^{\mp 2 n \pi}}{n^{2}}
\end{gathered}
$$


where in the last step we have substitute in the poles $s_{n}=\frac{n \pi}{g E_{0}}$ and simplify. The $(-1)^{n+1}$ comes from $-\cos (n \pi)$. The $e^{+n \pi}$ choice in (41) leads to a divergent $\gamma$ so we take the $e^{-n \pi}$ choice in (41) which leads to

$$
\begin{aligned}
\gamma=L^{3} T & \frac{g^{2} E_{0}^{2}}{16 \pi^{3}} \sum_{n=1}^{\infty}(-1)^{n+1} \frac{e^{-2 n \pi}}{n^{2}} \approx \\
& \approx 0.00187 \times L^{3} T \frac{g^{2} E_{0}^{2}}{16 \pi^{3}} .
\end{aligned}
$$

In the last step we have carried out the sum numerically with the result $\sum_{n=1}^{\infty}(-1)^{n+1} \frac{e^{-2 n \pi}}{n^{2}} \approx$ 0.00187 .

Calculations similar to the above were carried out in $[9,10,11]$ for gluon production in a constant $S U$ (3) color electric field. In these works the integration over the transverse momentum was not carried out, but if one does carry out the integration of the transverse momentum of the results in $[9,10$, 11] one finds that our result for $\gamma$ in (42) is consistent with these previous results. In particular there is no mass suppression (as expected) and the production rate is proportional to $g^{2} E_{0}^{2}$

Appendix II: Uniform Color Magnetic Field

\section{Calculation}

In this appendix we show that, unlike the uniform color electric field of appendix I, a uniform color magnetic field does not produce gluons. This is similar to what occurs in the electromagnetic Schwinger effect - a uniform magnetic field does not produce electrons/positrons.

A constant color magnetic field in the $\hat{z}=3$ spatial direction and in the $a=3$ color direction,

$$
\begin{aligned}
B_{\mu}^{a} \rightarrow & B_{3}^{3}=F_{12}^{3}=\partial_{1} A_{2}^{3}-\partial_{2} A_{1}^{3}+ \\
& +g \varepsilon^{3 b c} A_{1}^{b} A_{2}^{c}=H_{0} \hat{z},
\end{aligned}
$$

can be obtained by the potential

$$
A_{\mu}^{a}=-H_{0} x_{1} \delta_{\mu 2} \delta^{a 3}
$$

With the potential in (44) we perform a long calculation that is similar to the one for the constant color electric field, which leads to

$$
\left(\partial^{\mu}-i g H_{0} x \delta^{\mu 2}\right)^{2} W_{\nu}-2 g H_{0}\left(\begin{array}{llll}
0 & 0 & 0 & 0 \\
0 & 0 & i & 0 \\
0 & -i & 0 & 0 \\
0 & 0 & 0 & 0
\end{array}\right) W_{\mu}=0
$$

which is the color magnetic version of (29). Expanding (45) out we arrive at the color magnetic version (31) which leads to

$$
\left[\frac{\partial^{2}}{\partial t^{2}}-\frac{\partial^{2}}{\partial x^{2}}-\frac{\partial^{2}}{\partial z^{2}} \mp 2 g H_{0}-\left(i \frac{\partial}{\partial y}+g H_{0} x\right)^{2}\right] W_{v}=0 .
$$

Next we Fourier transform (46) to $\widetilde{W}_{v}\left(t, k_{1}, k_{2}, k_{3}\right)$ gives the color magnetic version of (32)

$$
\left[-E^{2}+k_{3}^{2} \mp 2 g H_{0}-\frac{\partial^{2}}{\partial x^{2}}+g^{2} H_{0}^{2}\left(x+\frac{k_{2}}{g H_{0}}\right)^{2}\right] \widetilde{W}_{v}=0 .
$$

Making the change of variable $\eta=x+\frac{k_{2}}{g H_{0}}$ this becomes

$\left[-E^{2}+k_{3}^{2} \mp 2 g H_{0}-\frac{\partial^{2}}{\partial \eta^{2}}+g^{2} H_{0}^{2} \eta^{2}\right] \widetilde{W}_{v}=0$.

The $-\frac{\partial^{2}}{\partial \eta^{2}}+g^{2} H^{2} \eta^{2}$ part of the above equation is the equation for a simple harmonic oscillator which has eignevalues $2\left(n+\frac{1}{2}\right) g H_{0}$. Substituting this eigenvalue into (48) leads to

$$
\left[-E^{2}+k_{3}^{2} \mp 2 g H_{0}+2\left(n+\frac{1}{2}\right) g H_{0}\right] \widetilde{W}_{v}=0 .
$$

We now rotate to imaginary time (Euclidean) as $t \rightarrow-i \tau$ and in conjunction with this we rotate the energy as $E \rightarrow=-i E$. This turns (49) into 
$\left[E^{2}+k_{3}^{2} \mp 2 g H_{0}+2\left(n+\frac{1}{2}\right) g H_{0}\right] \widetilde{W}_{v}=0$.

The eigenvalues for the system can then be written out as

$$
\lambda_{n}=E^{2}+k_{3}^{2} \mp 2 g H_{0}+2\left(n+\frac{1}{2}\right) g H_{0} .
$$

Now substituting (51) into (5) yields $\zeta$ for this case as

$$
\zeta=-\sum_{n} \int_{0}^{\infty} \frac{\mathrm{d} s}{s} \exp \left\{-\left[E^{2}+k_{3}^{2}+(2 n+1) g H_{0} \mp 2 g H_{0}\right] s\right\} .
$$

Recalling that we are quantizing in a cube with time $\tau=-i T$ transforms (52) into sides length $L$ and over a total (imaginary) interaction

$$
\zeta=-\tau \int_{-\infty}^{\infty} \frac{\mathrm{d} E}{(2 \pi)} L \int_{-\infty}^{\infty} \frac{\mathrm{d} k_{3}}{(2 \pi)} L^{2} \frac{g H_{0}}{2 \pi} \sum_{n=0}^{\infty} \int_{0}^{\infty} \frac{\mathrm{d} s}{s} \exp \left\{-\left[E^{2}+k_{3}^{2}+(2 n+1) g H_{0} \mp 2 g H_{0}\right] s\right\} .
$$

The $E$ and $k_{3}$ integrations are simple Gaussian integrals that give two factors of $\sqrt{\frac{\pi}{s}}$. Thus (53) simplifies to

$$
\zeta=-\tau L^{3} \frac{g H_{0}}{8 \pi^{2}} \sum_{n=0}^{\infty} \int_{0}^{\infty} \frac{\mathrm{d} s}{s^{2}} \exp \left\{-\left[(2 n+1) g H_{0} \mp 2 g H_{0}\right] s\right\}
$$

We now use the geometric series $\sum_{n=0}^{\infty} e^{-(2 n+1) g H s}=\frac{1}{2} \frac{1}{\sinh (g H s)}$ to write (54) as

$$
\zeta=-\tau L^{3} \frac{g H_{0}}{16 \pi^{2}} \int_{0}^{\infty} \frac{\mathrm{d} s}{s^{2}} \frac{e^{ \pm 2 g H_{0} s}}{\sinh \left(g H_{0} s\right)} .
$$

We now return to real time via the rotation $\tau \rightarrow i T$.

$$
\zeta=-i T L^{3} \frac{g H_{0}}{16 \pi^{2}} \int_{0}^{\infty} \frac{\mathrm{d} s}{s^{2}} \frac{e^{ \pm 2 g H_{0} s}}{\sinh \left(g H_{0} s\right)} .
$$

In contrast to the color electric case of the the previous appendix where the rotation back to real time, $\tau \rightarrow i T$, was accompanied by a rotation of the electric field amplitude, $E_{0} \rightarrow i E_{0}$, here the rotation back to real time does not lead to a change in the color magnetic field amplitude. In comparing (56) with (39) one finds that (56) does not have the series of poles at $s_{n}=n \pi / q E_{0}$ that are found in (39). Therefore the integral in (56) does not have any imaginary contributions coming from the residue theorem as was the case for the integral in (39). Thus from (56) the real part of $\zeta$ is zero so $\operatorname{Re}(\zeta)=\gamma=0$. Thus in the case of a uniform color magnetic field there is no particle production as is expected form the electromagnetic case where one finds that a constant magnetic field does not produce electron/positron pairs.

\section{References}

1. J. Schwinger. On Gauge Invariance and Vacuum Polarization // Phys. Rev. - 1951. - Vol. 82. - P. 664-679.

2. W. Heisenberg, H. Euler. Consequences of Dirac Theory of the Positron // Z. Physik. - 1936. - Vol. 98. - P. 714.

3. B. R. Holstein. Strong field pair production // Am. J. Phys. - 1999. - Vol. 67. - P. 499-507.

4. K. Srinivasan, T. Padmanabhan. Particle production and complex path analysis // Phys. Rev. - 1999. - D60. - P. 024007.

5. R. Brout, R. Parentani, Ph. Spindel. Thermal-properties of pairs produced by an electric-field - a tunneling approach // Nucl. Phys. - 1991. - B353. - P. 209-236.

6. F. Gelis, N. Tanji. Schwinger mechanism revisited // Prog. Part. Nucl. Phys. - 2016. - Vol. 87. - P. 1-49.

7. B.R. Holstein. Topics in Advanced Quantum Mechanics // Addison-Wesley. Reading. MA. - 1992. Chap. III.6.
8. W. Greiner, A. Schäfer. Quantum Chromodynamics // Springer-Verlag. Berlin. - 1994. Chap. 8.1.

9. G. C. Nayak, P. van Nieuwenhuizen. Soft-gluon production due to a gluon loop in a constant chromoelectric background field // Phys.Rev. D. - 2005. Vol. 71. - P. 125001.

10. F. Cooper, G. C. Nayak. Nonperturbative gluon pair production from a constant chromo-electric field via the Schwinger mechanism in arbitrary gauge // Phys.Rev. D. - 2006. - Vol. 73. - P. 065005.

11. G. C. Nayak. Schwinger Mechanism for Gluon Pair Production in the Presence of Arbitrary Time Dependent

12. F. D. Aaron et. al. Measurement of dijet production in diffractive deep-inelastic scattering with a leading proton at HERA // Eur. Phys. J. C. - 2009. - Vol. 59. - P. 715. 